BLS 33, No 1 2007. DOI: http://dx.doi.org/10.3765/bls.v33i1.3520

(published by the Berkeley Linguistics Society and the Linguistic Society of America)

\title{
The Interaction of Tones and Vowels in Fuzhou
}

\author{
CATHRYN DONOHUE \\ Australian National University
}

\section{Introduction}

The goal of this paper is to investigate the coincident changes of tones and vowels in Fuzhou. There has been considerable work on understanding the phonetic effects of segments on tone and on the influence of tone on segments (e.g. Maddieson 1977, 1984, 1997, Zee 1984 and others). Such phonetic effects are often the consequence of speech production and as such are relatively imperceptible. However, less attention has been given to effects of tone and segments that are not automatic phonetic changes, but rather changes that result from the phonology. In this paper I present data from the Fuzhou dialect of Chinese. I argue that the phonological tone is responsible for the segmental effects, rather than the reverse, but that the phonological segments may nonetheless play a role in the tonal outputs. I argue this point using acoustic data from Fuzhou.

\section{Fuzhou}

Fuzhou is a Min dialect of Chinese spoken in north-eastern Fujian province, China. There are seven citation tones in Fuzhou, but previous work differs enormously on the actual shape of the contours. Table 1 presents the tonal representations available in the literature. The contour descriptions are given using the Chao tone letters, which typically uses a scale of $1-5$, where 5 is the highest and 1 is the lowest pitch. The underlining indicates that the tone occurs with a 'stopped' syllable, which in Fuzhou implies a glottal stop coda, found with tones 4 and 7.

In this table we see a lot of minor variation, such as the exact height of tone 1, which is clearly a high level tone. But there is more worrying variation for other tones, such as tone 2 , which is possibly a mid or low tone, and is level or falling. Tone 3 is represented as a mid-rise, a dipping tone, a low-rise, a low level, and a mid-fall. Tone 4 is always a low(ish) rising tone (ending in the glottal stop), and tone 5 is clearly a high falling tone. Tone 6 is a convex tone, but varies between low, mid and high rise-fall. Tone 7 is clearly a high tone (with a glottal closure), but how high it is and whether there is a slight rise is not clear. 


\section{Cathryn Donohue}

Table 1. Previous descriptions of Fuzhou tones.

\begin{tabular}{lcccccccc}
\hline Author & Year & Tone 1 & Tone 2 & Tone 3 & Tone 4 & Tone 5 & Tone 6 & Tone 7 \\
\hline Chen & 1985 & 44 & 32 & 213 & $\underline{13}$ & 51 & 131 & $\underline{5}$ \\
Chen & 1967 & 44 & 22 & 312 & $\underline{24}$ & 52 & 232 & $\underline{5}$ \\
Corbato & 1945 & 44 & 21 & 25 & $\underline{24}$ & 52 & 232 & $\underline{5}$ \\
Ergerod & 1956 & 55 & 33 & 13 & $\underline{13}$ & 52 & 242 & $\underline{55}$ \\
Lan & 1953 & 55 & 33 & 11 & $\underline{13}$ & 61 & 242 & $\underline{56}$ \\
Jiang-King & 1999 & 44 & 31 & 213 & $\underline{23}$ & 53 & 242 & $\underline{5}$ \\
Nakajima & 1979 & 55 & 33 & 31 & $\underline{23}$ & 52 & 242 & $\underline{55}$ \\
Norman & 1988 & 55 & 22 & 13 & $\underline{24}$ & 41 & 342 & $\underline{55}$ \\
Tao & 1930 & 55 & 31 & 13 & $\underline{\underline{34}}$ & 52 & 342 & $\underline{\mathbf{5}}$ \\
Yip & 1980 & 44 & 22 & 12 & $\underline{\underline{13}}$ & 52 & 242 & $\underline{4}$ \\
Yuan & 1980 & 44 & 31 & 213 & $\underline{23}$ & 52 & 353 & $\underline{4}$ \\
\hline
\end{tabular}

This sort of variation is conceivably dialectal variation or between-speaker variation, but it certainly illustrates the need to work from quantified data. In Table 2, I have summarized the Fuzhou tones impressionistically, and in Section 4 I present Donohue (1992a)'s normalized F0 contours for Fuzhou tones.

Table 2. An impressionistic description of the Fuzhou tones.

\begin{tabular}{ll}
\hline Tone 1 & High level with a slight rise \\
Tone 2 & Mid level with a slight fall \\
Tone 3 & Low level with a slight fall \\
Tone 4 & Low rising stopped tone (final glottal stop) \\
Tone 5 & High fall, starting higher than the high level tone \\
Tone 6 & Low rise-fall \\
Tone 7 & High stopped tone (final glottal stop), notably shorter than the others \\
\hline
\end{tabular}

\section{Tone-vowel interaction}

The tone-vowel interaction in Fuzhou has to do with changes internal to a phonological vowel that are coincident with tonal changes. The phonological vowels are determined based on what are historically the same vowel and what are synchronically realized as the same vowel in other dialects of Chinese. The vowels are often described as 'high' and 'low', and the vowel pairs are illustrated in Table 3. 
Table 3. Examples of alternating vowel pairs in Fuzhou.

\begin{tabular}{|c|c|}
\hline Set A: Tones $1,2,5,7$ & Set B: Tones 3, 4, 6 \\
\hline $\mathrm{i}$ & ei \\
\hline ei & ai \\
\hline $\mathrm{u}$ & ou \\
\hline ou & $\mathrm{au}$ \\
\hline $\mathrm{y}$ & øу \\
\hline Øу & $\theta y$ \\
\hline $\mathrm{a}$ & $\mathrm{a}$ \\
\hline
\end{tabular}

Fuzhou tone sandhi is right dominant, which means that it is the final syllable in a given domain that remains unchanged. All the prepausal syllables retain their citation tones and vowels; however, in sandhi (non-final) position, the tone changes and the vowel also changes. Consider the examples in (1)-(3) below. The first two sets of examples illustrate that when a syllable with an underlying tone from 'Set B' (tone 3, 4,6) is in prepausal position or alone (the (a) examples), the 'lower' of the vowel pairs - its so-called underlying vowel - is realised. However, in sandhi position, we see that not only has the tone changed, the vowel has also changed to the 'higher' variant from Set A. (3) illustrates that the vowel in a syllable from the 'set A' tones $(1,2,5,7)$ remains unchanged in sandhi position.

(1) Tone 6: [tou]

T6 + T6: [tu 42 kei 231]

(2) Tone 3: [khei] $\mathrm{T} 3+\mathrm{T} 4: \quad[$ khi 52 a? $\underline{13}]$

(3) Tone 7: [tu $\underline{5}]$

T7 + T6: [tu 33 tau 231] 'think'

'imagine'

'air'

'air pressure'

'poison'

'poison bean'

It is worth noting that vowel height is clearly not the key difference between these two sets of vowels, especially when you have a pair of vowels: [ou]-[au], with [ou] in Set A and [au] in Set B. The difference looks somewhat like monophthong/diphthong pairings, but this characterization clearly won't account for all vowels, given the monophthongal [a] that remains the same between groups. Let us turn to phonological accounts for these vowel alternations, by discussing two previous works that have addressed this issue in some depth.

\subsection{Yip 1980}

Yip (1980) is an extensive study of tonal phonological phenomena in a range of Chinese languages. Her analysis of Fuzhou is that the Set B vowels are raised when they co-occur with tones that are [+upper] register. To account for this, she provides a rule, shown in (4). 


\section{Cathryn Donohue}

$$
\mathrm{V}[\alpha \text { low }] \rightarrow[- \text { low, }- \text { - } h \text { high }] /[+ \text { upper }]
$$

This elegant rule, however, fails to capture the simplification of diphthongs, nor does it readily account for the fact that some vowels, such as [a], are constant in ALL tonal contexts. Another shortcoming of this approach is that it results in a rather unusual vowel inventory, lacking $[\mathrm{i}, \mathrm{y}, \mathrm{u}]$, which are all necessarily considered derived vowels in this approach.

\subsection{Jiang-King 1999}

Jiang-King (1999) presents a study of the tone/vowel interaction in Northern Min, including Fuzhou. Jiang-King, following Hyman (1988 and elsewhere) includes in her tonal representation the moraic structure of the syllable, and relates the vowel alternations to the tonal changes by appealing to the syllable weight. She refers to the difference, not in terms of 'height' as Yip did, but rather in terms of 'tightness', whereby the Set A vowels are considered 'tight syllables' and the Set B vowels, 'loose'. Jiang-King's 'prosodic anchor hypothesis' (p. 77) captures this covariation by postulating, relatively noncontroversially, that tight syllables have one mora, while loose syllable have two. The leftmost mora is considered the head, and it can host up to two tones, while non-head morae can host only one tone. This captures the bigger picture that is Jiang-King thesis, that the moraic structure determines tone/vowel possibilities. There are other rules included to change the actual quality of the vowel.

This is a very neat proposal, drawing nicely on previous typologically tested work to inform the hypothesis, but runs into difficulty when accounting for vowels whose form, unlike, e.g. the $[y]-[\varnothing y]$ pair, does not change according to the moraic structure of the syllable consistent diphthongs in a pair (e.g. [ei]-[ai]), or the monophthong [a] noted above.

In contrast to these two proposals, I claim that there is no need to predict the actual phonetic shape of the vowel, just the phonological shift. I also claim that the reason for this shift is perceptual enhancement, found elsewhere in Fuzhou. The next sections present new data for Fuzhou that has been quantified and normalized across speakers, before discussing specifically the factors involved in perceptual enhancement in Fuzhou, including the tone/vowel alternations.

\section{Quantified Fuzhou data}

This section presents Fuzhou data from Donohue (1992a, forthcoming-a) taken from a single variety that has been quantified and normalized across four speakers (two male, two female).

Using three sets of tones with $[\mathrm{i}, \mathrm{a}, \mathrm{u}]$ as their nuclei, Donohue measured 3 tokens per vowel set, with two repetitions per token, going through the sets 3 times each in a randomized order (18 tokens per vowel set per speaker, which is 54 tokens per vowel per speaker). The tonal contours were then normalized across speakers using a z-score transform (Rose 1987). To try to limit sources of error, 
only syllable types with voiceless unaspirated plosive onsets were used, without nasal codas.

\subsection{Tones}

The normalized F0 contours for each of the seven citation tones are given below on the left hand side of Figure 1. The right hand side of the figure shows the normalized tone contours after determining that there were onset and offset perturbations, which were found to be $50 \mathrm{msecs}$ and $5 \%$ of the tonal duration, respectively.
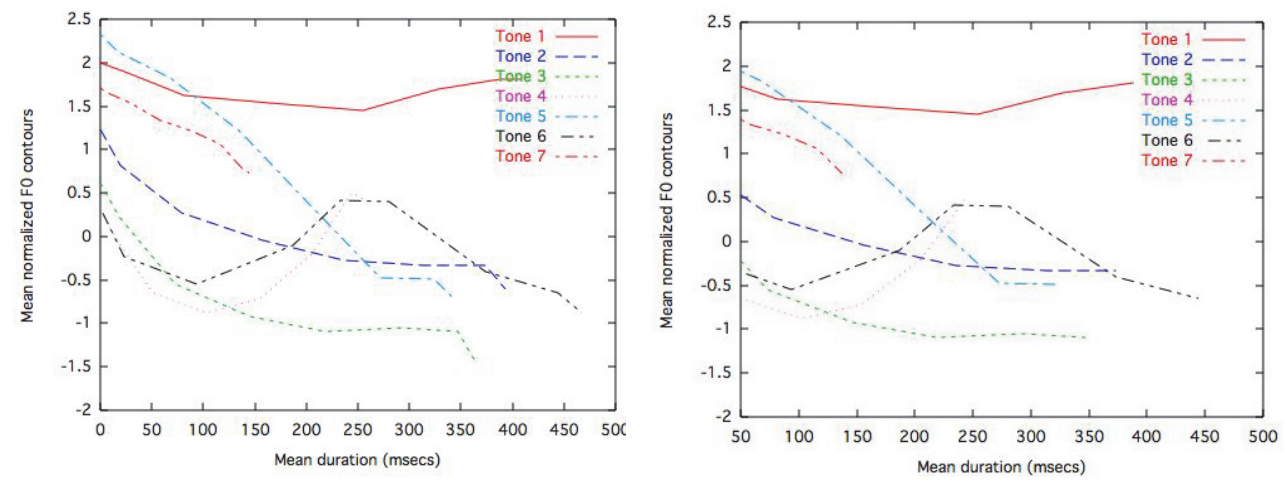

Figure 1. Left: Normalized tones. Right: Normalized tones in Fuzhou without onand off-set perturbations.

To represent these tones phonologically, I will use the reasonably standard features for register ([ \pm upper $]$ ) and tonal contour features ([ \pm high] (e.g. Bao 1990, Snider 1999). There is some question of the definition of register, but following Donohue (1992b), I assume that it is a bifurcation of the pitch range. As such, assigning tonal features to the contours is relatively straightforward, with the exception of tone 2: should it be considered a high tone in the lower register or a low tone in the higher register, given that it starts just above the mid-range point and drops into the lower register. If one were to assume that it is a [-upper] register, [+high] tone, then one could say that lower register tones fall slightly, as tone 3 does as well, whereas upper register level tones rise slight (like tone 1). However, we could also describe this as maximally distinguishing not the register features but the tonal features, thus a [+upper] register [-high] tone, and claim that all [-high] tones fall slightly, while [+high] tones rise slightly. I will assume that it is a [+upper, -high] tone, as it is then grouped with the other [+upper] tones forming a natural class for the Set A vowels. The feature assignment for the tones that I will assume is given in Table 4. The abbreviations use upper case for register features and lower case for tonal contour features. 


\section{Cathryn Donohue}

Table 4. Tonal feature assignment in Fuzhou.

\begin{tabular}{lll}
\hline Tone & Features: Register, Tone & Abbreviated features \\
\hline Tone 1 & [+upper], [+high] & $\mathrm{H}, \mathrm{h}$ \\
Tone 2 & [+upper], [-high] & $\mathrm{H}, \mathrm{l}$ \\
Tone 3 & [-upper], [-high] & $\mathrm{L}, 1$ \\
Tone 4 & [-upper], [-high, +high] & $\mathrm{L}, \underline{\mathrm{lh}}$ \\
Tone 5 & [+upper], [+high, -high] & $\mathrm{H}, \mathrm{hl}$ \\
Tone 6 & [-upper], [-high, +high, -high] & $\mathrm{L}, \mathrm{lhl}$ \\
Tone 7 & [+upper], [+high] & $\mathrm{H}, \underline{\mathrm{h}}$ \\
\hline
\end{tabular}

\subsection{Phonation}

Another finding of Donohue (1992a) is that Fuzhou has a consistent non-modal phonation change coincident with tones 3, 4 and 6-ALL THE LOWER REGISTER TONES. Tone 2 also shows some non-modal phonation, suggesting that perhaps it could have to do with the syllable starting with [-high]. These are summarized in Table 5.

The phonation changes consistently to a creaky voice in the lower register, but tone 2 also shows some deviation from modal phonation. I claim that this change in phonation is to perceptually enhance the change in tonal features used, with the lower register [-high] onset tones being produced in all creak, and the upper register [-high] getting only a slight breathiness.

Table 5. Phonation changes in Fuzhou.

\begin{tabular}{ll}
\hline Tone & Phonation \\
\hline Tone 1 & modal \\
Tone 2 & slightly breathy \\
Tone 3 & breathy/creaky voice \\
Tone 4 & creaky voice \\
Tone 5 & modal \\
Tone 6 & creaky voice \\
Tone 7 & modal \\
\hline
\end{tabular}

\subsection{Vowel quality}

It is worth noting that the same vowel can occur in both Sets A and B. Consider the pairs previously presented (Set A-Set B), we have [i]-[ei] and [ei]-[ai]. We also observe $[\mathrm{u}]-[\mathrm{ou}]$ and $[\mathrm{ou}]-[\mathrm{au}]$. Moreover, the monophthong is consistent across all tones. Understanding the vowel alternations is thus not a question of which vowel type is more suited to the moraic structure of the tone, or which are 'lower' or 'higher' in the relevant register. My claim is that it is a question of having the perceptual enhancement of the upper register through these regular 
vowel alternations, not a question of deriving the vowel alternations based on dynamic tonal assignment or tone/moraic structure.

\subsection{Tone sandhi}

To justify the claim that the vowel alternations and phonation changes serve to perceptually enhance the tone, it is necessary to explain that the full tonal paradigm and coincident changes of phonation and vowels only occur in citation/isolation form. That means that in sandhi position you find neither the vowel changes nor the phonation changes. This is perhaps not surprising, as sandhi tones are often only two-thirds the duration of non-sandhi tones. That is, non-sandhi tones, which are at the end of the domain in Fuzhou, or prepausal position, can be up to 1.5 times longer in duration. This is clearly a context in which it is much more feasible to enhance the perception of the tones by adding

the vowel and phonation cues. For historical origins of the vowel forms, see Donohue Forthcoming-b.

\section{Concluding remarks}

The main point of this paper is to claim that the vowels and tones are not dynamically interacting, but rather are merely co-occurring in a context that is seen to be a good one for perceptual enhancement. The Set B vowels indicate [-upper] register, and the phonation indicates a tone starting with a [-high] feature.

\section{References}

Chan, Marjorie. 1985. Fuzhou Phonology: A non-linear analysis of tone and stress. Ph.D. dissertation, University of Washington.

Chen, Leo. 1967. Foochow reduplication. Tsing Hua Journal of Chinese Studies 6:200-213.

Corbato, Hermenegildo (ed.). 1945. Manual of the Foochow dialect. Los Angeles: UCLA.

Donohue, Cathryn. 1992a. The phonetics and phonology of Fuzhou tones. Honours thesis, Australian National University.

Donohue, Cathryn. 1992b. The phonetics of register in the Fuzhou dialect of Chinese. Proceedings of the Fourth Australian International Conference on Speech Science and Technology, 602-607. Brisbane: University of Queensland Press.

Donohue, Cathryn. Forthcoming-a. Fuzhou tonetics and tonology. Munich: Lincom Europa.

Donohue, Cathryn. Forthcoming-b. Tones and vowels in Fuzhou revisited. In Wolfgang Kehrein and Björn Köhnlein (eds), Tone and Segmental Structure. Amsterdam: Niemeyer Linguistische Arbeiten.

Ergerod, Søren. 1956. The Lungtu dialect: A descriptive and historical study of a south Chinese idiom. Copenhagen: Ejnar Munksgaard. 


\section{Cathryn Donohue}

Hyman, Larry M. 1988. Syllable structure constraints on tonal contours. Linguistique Africaine 1:49-60.

Jiang-King, Ping. 1996. An optimality account of tone-vowel interaction in northern Min. PhD dissertation, University of British Columbia.

Lan, Ya-Shiu. 1953. Phonetic system of Foochow. Bulletin of the College of Arts, National Taiwan University 5:241-331.

Maddieson, Ian. 1977. Tone effects on consonants. University of California Working Papers in Phonetics 36:91-110.

Maddieson, Ian. 1984. The effects on F0 of a voicing distinction in sonorants and their implications for a theory of tonogenesis. Journal of Phonetics 12(1):915.

Maddieson, Ian. 1997. Phonetic universals. In W. Hardcastle and J. Laver, eds., Handbook of Phonetic Sciences, 619-639. Oxford, Blackwell Press.

Nakajima, Motoki. 1979. A comparative lexicon of Fukien dialects. Ajia-Afurika Gengo Bunka Kenkyu (Research in Asian-African Language and Culture) 18:220-245.

Norman, Jerry. 1988. Chinese. Cambridge, Cambridge University Press.

Rose, Philip. 1987. Considerations in the normalisation of the fundamental frequency of linguistic tone. Speech Communication 6(4):343-352.

Snider, Keith. 1999. The geometry and features of tone. Dallas, Dallas SIL and University of Texas, Arlington.

Tao, Yu-Min. 1930. Phonetics of the Foochow dialect. Minyin Yanjin 1(4):445470.

Yip, Moira. 1980. The tonal phonology of Chinese. $\mathrm{PhD}$ dissertation, Massachusetts Institute of Technology.

Yuan, Jia-Hua. 1980. Hanyu Fangyan Gaiyao (A study of Chinese dialects). Beijing, Wenzi Gaige Chubanshe.

Zee, Eric. 1984. Peak intraoral air pressure in [p] as a function of F0 in Chinese. Language and Speech 27(4):381-390.

Zhiming, Bao. 1990. On the nature of tone. PhD dissertation, Massachusetts Institute of Technology.

Department of Linguistics

College of Asia \& the Pacific

Australian National University

Canberra 0200 AUSTRALIA

cathryn@donohue.cc 